\title{
COMPARISON OF THE MODELS OF THE AIR GAUGE STATIC CHARACTERISTICS
}

\begin{abstract}
In the article, the authors analyze and discuss several models used to the calculation of air gauge characteristics. The model based on the actual mass flow (which is smaller than the theoretical one) was proposed, too. Calculations have been performed with a dedicated software with the second critical parameters included. The air gauge static characteristics calculated with 6 different models were compared with the experimental data. It appeared that the second critical parameters model (SCP) provided the characteristics close to the experimental ones, with an error of ca. $3 \%$ within the air gauge measuring range.
\end{abstract}

\section{Introduction}

The very first documented attempt to describe scientifically the work of the air gauge was undertaken as early as in 1932 by Mennesson [1]. Since then, many scientists presented various concepts of analytical representation of the air gauge behavior in order to predict its metrological properties and calculate its geometry, e.g. $[2,3]$. Though measurement science and instrumentation developed enormously since then, in many cases the air gauges may be not replaced by other measuring devices. It is so mostly because of their low sensitivity to the environmental conditions and ability to perform non-contact measurements [4]. In checking any dimensional characteristic, air gauge offers sufficient magnification and reliability to measure tolerances well beyond the scope of mechanical gauges [5]. In the last

\footnotetext{
${ }^{1}$ Poznan University of Technology, Institute of Mechanical Technology, Division of Metrology and Measurement Systems, Piotrowo 3, 60-965 Poznan, Poland; Email: czeslaw.jermak@put.poznan.pl,

${ }^{2}$ Poznan University of Technology, Chair of Thermal Engineering, Piotrowo 3, 60-965 Poznan, Poland

${ }^{3}$ Faculty of Mechanical Engineering, Kazimierz Pulaski University of Technology and Humanities in Radom, 54 Krasickiego Str., 26-600 Radom,Poland; Email: m.rucki@uthrad.pl
} 
decade, rapid improvement may be noted in the air gauging because of application of various electronic devices and digital signal processors [6].

On the one hand, measurement science has become closely associated with computer, information, control and systems science [7]. Incorporated into the industrial measurement system, air gauges produce a signal processed by advanced digital systems [8]. The rapid development of computers and numerical calculation made it possible to use more precise and more sophisticated methods for data processing, which was too complicated to be conducted in the past [9]. Thus, more accurate models of the air gauge are required.

On the other hand, most of the existing models do not stay in conformity with measurement data and should be corrected with data obtained from experiments [10]. It indicates the need of accurate and reliable experimental setup to check the proposed theoretical models.

Knowing the static characteristics, which are the functions of the back-pressure dependent on slot width $p_{k}=f(s)$, one can predict the metrological characteristics of the air gauge, such as measuring range $z_{p}$ and multiplication $K$. The function should take into consideration such important factors as geometry of the nozzles and their true flow characteristics. The present work discusses some typical mathematical models of the static characteristics of back-pressure air gauges and their accuracy.

\section{The measuring chamber and the airflow conditions in it}

The air gauge is fed by pressured air of the absolute pressure pz(a). In the inlet nozzle with the orifice of diameter $d_{w}$, the air stream velocity acceleration takes place, which results in the pressure fall [11]. After leaving the area of inlet nozzle marked in the Fig. 1 as (I), the air stream expands creating the secondary flow [12], and after some distance it occupies the entire chamber (in its diameter $d_{k}$ ). Outside the main stream, whirls and back-streams appear in the secondary flow. Moreover, the backflow reduces the cross-sectional area through which the fluid flows [13]. After the air stream expands inside the chamber, its pressure $p_{k}$ could be measured with the pressure transducer of any type. This value is called the back-pressure and it is used for indirect measurement of the flapper surface distanced from the measuring nozzle with the slot $s$ (Fig. 1).

The fall of air pressure inside the measuring chamber down to pk value could be expressed by the pressure loss coefficient $\zeta_{k t}$. The air flows through the measuring nozzle of diameter $d_{p}$ (II) and its expansion inside the slot $\mathrm{s}$ results in further fall of pressure. In the flapper-nozzle area, the radial expansion takes place, which has been described in several publications, e.g. [14]. Here, the coefficient $\zeta_{z}$ should be introduced, which bounds the pressure in the measuring nozzle with the atmospheric pressure $p_{a}$. 


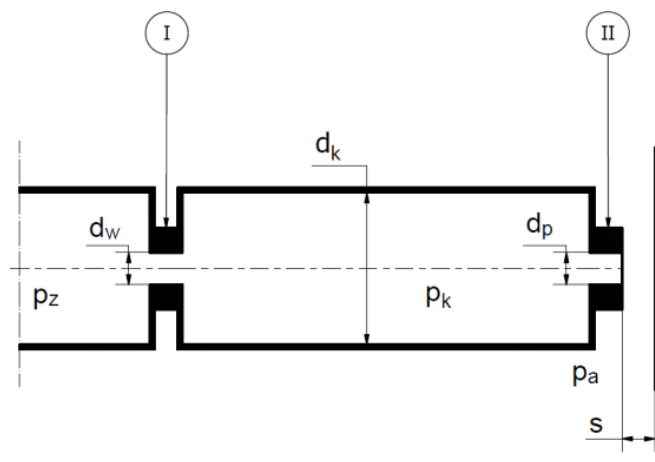

(a)

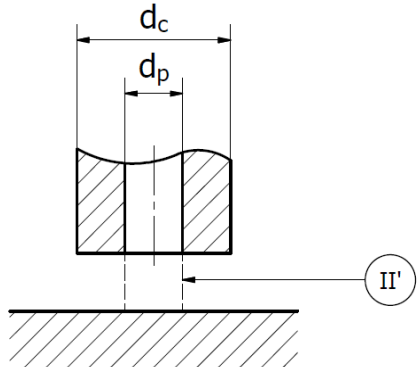

(b)

Fig. 1. Air gauge scheme: a) inlet nozzle I and measuring nozzle II, b) outflow area in the measuring slot II'

The stream contraction causes certain losses of pressure, therefore the actual pressure ratio for maximal mass flow differs from the theoretical one. It is called the second critical ratio $\beta_{k r 2}$ [15]. The corresponding flow coefficient is:

$$
\alpha_{k r 2}=\frac{\dot{m}_{r z \max }}{\dot{m}_{t \max }}
$$

where $\dot{m}_{r z \max }, \dot{m}_{t \max }$ represent actual and theoretical maximal mass flow, respectively.

The air flow coefficient $\alpha$ is defined as the relation of the actual mass flow to its theoretical value, and it represents the losses in the air flow. It could be written:

$$
\alpha=\alpha_{k r 2} \frac{q_{1}}{q_{2}}
$$

where:

$q_{1}=\frac{\dot{m}}{m_{r z \max }}-$ actual relative mass flow,

$q_{t}=\frac{\dot{m}_{t}}{m_{t} \max }-$ theoretical (isentropic) relative mass flow.

Another formula for the flow coefficient assuming steady flow could be written using the Sanville expression [16] or be derived as follows [17]:

$$
\alpha=\frac{1}{q_{t}} \frac{\alpha_{k r 2}}{1-\beta_{k r 2}} \sqrt{1-2 \beta_{k r 2}(1-\beta)-\beta^{2}}
$$

Even though the assumed simplification provided less accurate results for the pressure ratio $0.7 \leqslant \beta<1$, it may be applied because of easiness of calculation. Fig. 2 presents the example of the flow coefficient $\alpha$ obtained for different pressure ratios $p_{2} / p_{1}$. 


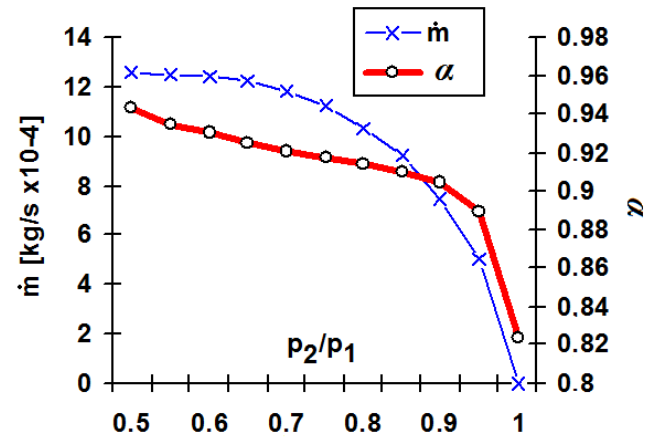

Fig. 2. Flow ratio $\alpha$ obtained for different pressure ratios $p_{2} / p_{1}$ [15]

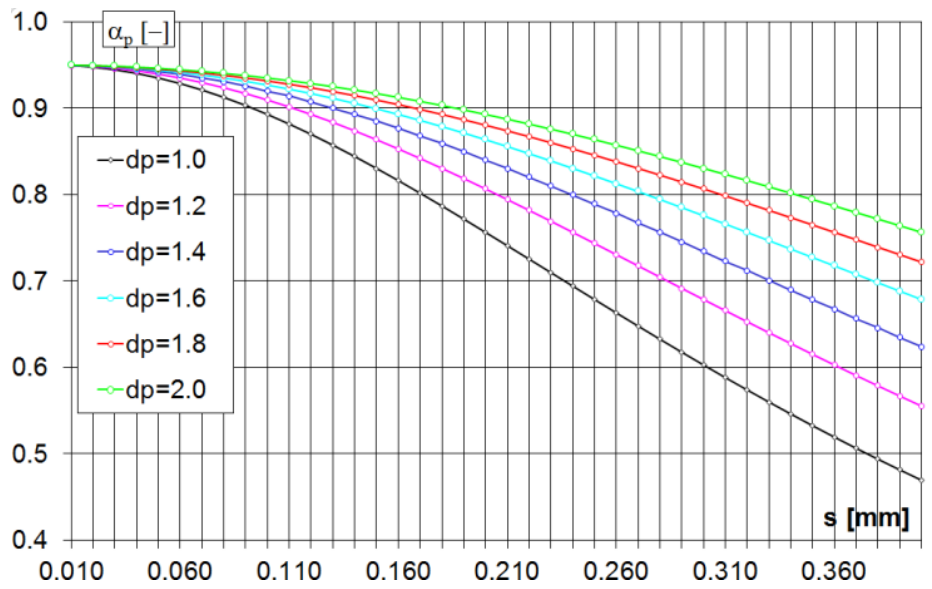

Fig. 3. Flow ratio $\alpha_{p}=f(s)$ in the flapper-nozzle area for different nozzles $d_{p}$

The values of the flow coefficient $\alpha$ and the critical flow ratio depend also on the shape of the flow-through channel [18]. When the air gauge works, the outflow depends on the slot width $s$, and hence, the outflow coefficient depends on it, too. Fig. 3 presents the values of flow coefficient in the flapper-nozzle area $\alpha_{p}$ versus the slot width $s$ for different diameters of the measuring nozzles $d_{p}$.

\section{Exact and simplified formulas of the flow in air gauges}

Airflow calculation appears to be a complicated task [19]. A number of mathematical models has been applied to calculate the static characteristics of the air gauges. Each of them is based on the formulas of the flow through the inlet nozzle and in the flapper-nozzle area $[20,21]$. The differences between the models lay basically in the loss coefficients and in the way of presenting the function itself.

The approach based on the Saint Venant - Wenzel's formulas distinguishes between different stages of the flow. Because the critical flow ratio is not reached 
simultaneously in areas I and II (Fig. 1), there are four possible combinations of the flow rates compared to the critical value [22]:

I) when in the inlet nozzle pressure ratio is $\beta_{w}>\beta_{k r 1}$ and in the flapper-nozzle area $\beta_{p}>\beta_{k r 1}$ as well,

II) when in the inlet nozzle $\beta_{w}<\beta_{k r 1}$ but in the flapper-nozzle area $\beta_{p}>\beta_{k r 1}$,

III) when in the inlet nozzle $\beta_{w}>\beta_{k r 1}$ but in the flapper-nozzle area $\beta_{p}<\beta_{k r 1}$,

IV) when in the inlet nozzle $\beta_{w}<\beta_{k r 1}$ and in the flapper-nozzle area $\beta_{p}<\beta_{k r 1}$ as well.

The condition of the mass flow continuity should be met considering the inlet nozzle $\dot{m}_{w}$ and the flapper-nozzle area $\dot{m}_{p s}$ :

$$
\dot{m}_{w}=\dot{m}_{p s}
$$

Table 1 presents the formulas applied to the above-listed flow rates combinations. The ratios of pressure $\beta_{w}$ ( $y$ in the Table 1$)$ and $\beta_{p}$ ( $b$ in the Table 1$)$, as well as effective flow diameter $x$ made it possible to avoid units in the final form. The latter is calculated as following:

$$
x=\frac{\alpha_{p}}{\alpha_{w}} \frac{A_{p s}}{A_{w}}
$$

Surfaces $A_{p s}$ and $A_{w}$ correspond with the air flow area in the measuring slot and in the inlet nozzle, respectively (areas II' and I in the Fig. 1. Similarly, flow coefficients $\alpha_{p}$ and $\alpha_{w}$ correspond with the losses in those two areas of the air gauge.

In most publications, the outflow surface $A_{p s}$ is considered to be a cylinder with its axis placed along the axis of the nozzle orifice, diameter equal to the nozzle diameter $d_{p}$, and the height equal to the slot width $s$ :

$$
A_{p s}=\pi d_{p} s
$$

It has been proved, however, that the geometrically minimal surface is different from the side cylinder surface [23]. It is a rather conical surface, as it is shown in Fig. 4 and described by the following equation:

$$
A_{s \min }=A_{p s} k\left(s_{w}\right)
$$

where:

$$
\begin{aligned}
& k\left(s_{w}\right)=\frac{1+k_{e}}{4} \sqrt{4+\left(\frac{1-k_{e}}{s_{w}}\right)^{2}}, \\
& s_{w}=\frac{s}{d_{p}}, \\
& k_{e}=0.5+0.5 \sqrt{1-8 s_{w}^{2}} .
\end{aligned}
$$




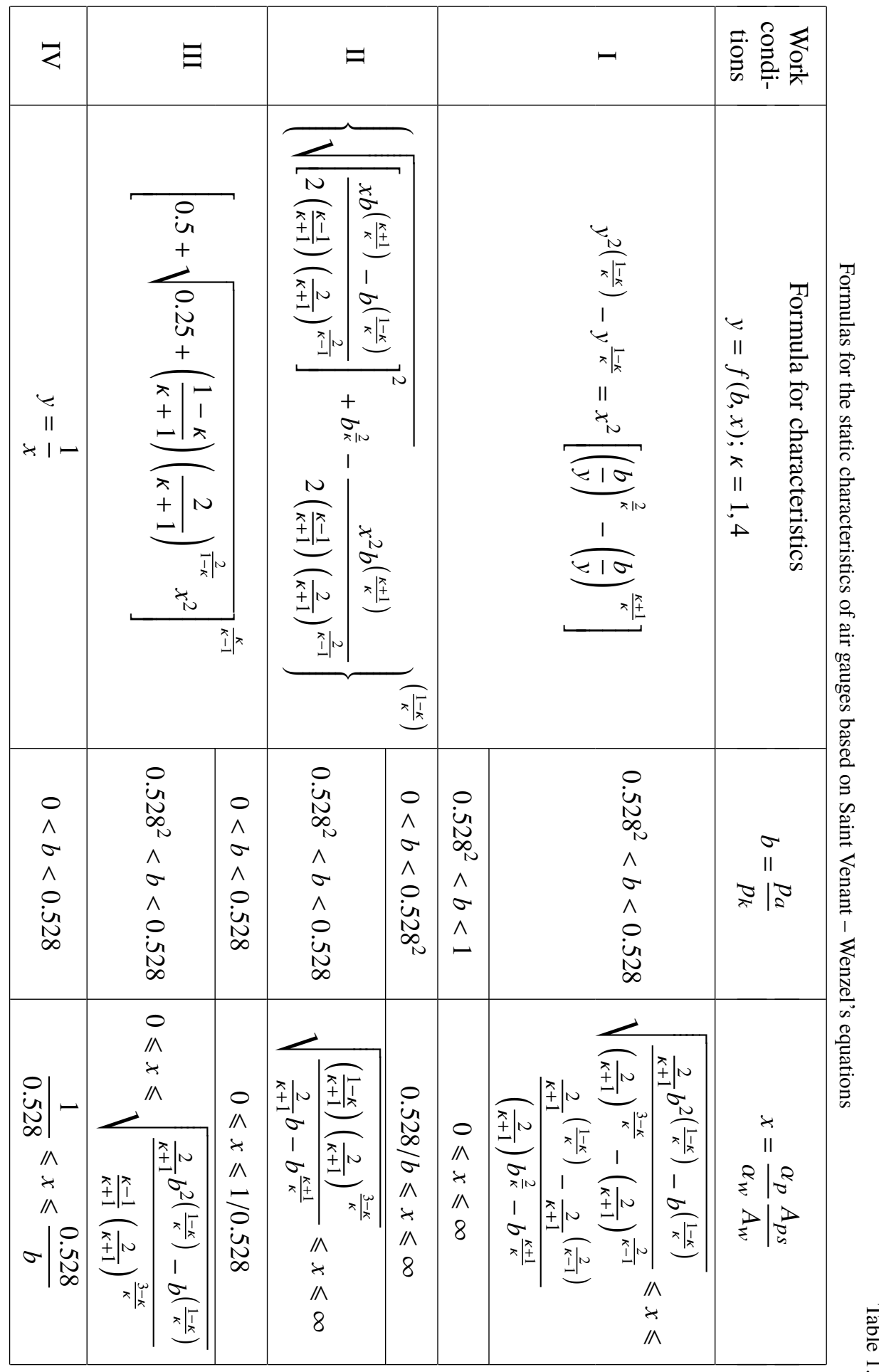




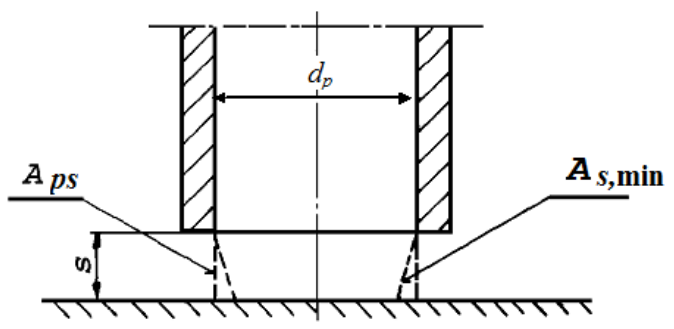

Fig. 4. Commonly calculated surface $A_{p s}$ and minimal surface $A_{s, \min }$ [23]

Fig. 4 presents the cylindrical surface $A_{p s}$ which is different from the minimal conic surface $A_{s}$ min. In accurate calculations, the latter should be considered a true outflow surface.

Consequently, the static characteristics calculated by using cylindrical and conical outlet surfaces are very different, especially in the measuring range of the air gauge.

\section{Simplified equations}

The exact formulas could be replaced with fairly accurate simplified ones [21]. For the pressure fall in the pressure ranges below the critical value, the following function could be applied:

$$
\dot{m}_{w}=A \sqrt{\frac{2}{R T_{z}} p_{z}\left(p_{z}-p_{1}\right)}
$$

and for the pressure ratios above the critical one:

$$
\dot{m}_{w}=A \sqrt{\frac{1}{2 R T_{z}} p_{z}}
$$

where: $A$ is the flow cross-section area, $R$ is the gas constant for air, $T_{z}$ is temperature of the feeding air, $p_{z}$ is feeding pressure, $p_{1}$ is pressure after the restriction.

For the practical purposes, from formulas (8) and (9) the simplified equations were derived to calculate the static characteristics of the air gauges [24]. In order to keep consistency with Table 1 , and to obtain unitless result, parameters $b$ and $x$ were introduced:

Earlier works proposed drastic simplification. For example, in [22] only cases I and III were taken into consideration, and three additional correcting factors $k$ were introduced:

$$
p_{k(a)}=\frac{p_{z(a)}}{1+16 k_{1} k_{2} k_{3} \frac{d_{p}^{2} s^{2}}{d_{w}^{2}}}
$$


Table 2.

Simplified equations for normalized static characteristics [24]

\begin{tabular}{|c|c|c|c|}
\hline $\begin{array}{l}\text { Work } \\
\text { condi- } \\
\text { tions }\end{array}$ & $\begin{array}{l}\text { Formula for characteristics } \\
\qquad y=f(b, x) ; \kappa=1,4\end{array}$ & $b=\frac{p_{a}}{p_{k}}$ & $x=\frac{\alpha_{p}}{\alpha_{w}} \frac{A_{p s}}{A_{w}}$ \\
\hline \multirow[t]{2}{*}{ I } & \multirow[t]{2}{*}{$y=\sqrt{\left(\frac{b x^{2}-1}{2}\right)^{2}+(b x)^{2}-\frac{b x^{2}-1}{2}}$} & $0.25 \leqslant b \leqslant 0.5$ & $\sqrt{\frac{2}{b}-4} \leqslant x \leqslant \frac{1}{2 \sqrt{\frac{b}{2}-b^{2}}}$ \\
\hline & & $0.5 \leqslant b \leqslant 1$ & $0 \leqslant x \leqslant \infty$ \\
\hline \multirow[t]{2}{*}{ II } & \multirow{2}{*}{$y=b+\frac{1}{b(2 x)^{2}}$} & $0 \leqslant b \leqslant 0.25$ & $\frac{1}{2 b} 0 \leqslant x \leqslant \infty$ \\
\hline & & $0.25 \leqslant b \leqslant 0.5$ & $\frac{1}{2 \sqrt{\frac{b}{2}-b^{2}}} 0 \leqslant x \leqslant \infty$ \\
\hline \multirow{2}{*}{ III } & \multirow{2}{*}{$y=\frac{1}{1+\left(\frac{x}{2}\right)^{2}}$} & $0 \leqslant b \leqslant 0.25$ & $0 \leqslant x \leqslant 2$ \\
\hline & & $0.25 \leqslant b \leqslant 0.5$ & $0 \leqslant x \leqslant \sqrt{\frac{2}{b}-4}$ \\
\hline IV & $y=\frac{1}{x}$ & $0 \leqslant b \leqslant 0.25$ & $0 \leqslant x \leqslant \frac{1}{2 b}$ \\
\hline
\end{tabular}

Index $(a)$ means that the pressure is in absolute values. The factors $k_{1}, k_{2}$ and $k_{3}$ correspond with the changes in stream velocity and mass flow caused by the variation of viscosity value and flow coefficients. They are presented in Table 3.

However, omitting the air compressibility leads to a substantial error. It may reach almost 30\% for the Mach number $M=2$ [22]. Moreover, the investigations proved that such a simplification assuming $\frac{\alpha_{p}}{\alpha_{w}}=1$ could lead to the errors of $\pm 20 \%$ and more, especially in the range of small slots. Fig. 5 presents several graphs of the $\frac{\alpha_{p}}{\alpha_{w}}$ ratio, which in the range of slots $s$ from 100 to $450 \mu \mathrm{m}$ vary from 0.8 up to 1.2 , dependent on the inlet nozzle diameter. For smaller slot widths, the ratio differs more for different $d_{w}$, reaching even 1.9 in the case of $d_{w}=1.805 \mathrm{~mm}$ and the slot width $s=25 \mu \mathrm{m}$.

In [25], the proposed calculations were extremely simplified by replacing all the formulas with one simple function and all the coefficients with one value of $\alpha$. The latter could vary for different diameters of inlet nozzle, as it is shown in Table 4.

\section{Accuracy analysis for simplified and exact formulas}

The accuracy analysis was based on the experimental results. Two sets of the nozzles were used in the experimental check of the presented above formulas. The measuring nozzle of diameter $d_{p}=2.100 \mathrm{~mm}$ and outer diameter ratio $d_{c} / d_{p}=4$ was combined with two inlet nozzles: $d_{w}=1.002 \mathrm{~mm}$ and $d_{w}=1.805 \mathrm{~mm}$. The laboratory set for static characteristics analysis consisted of:

1. initial air pressure reducer LPR type made by FESTO, completed with a filter and a valve, 
Factors for the simplified equation (10) [22]

\begin{tabular}{|c|c|c|c|}
\hline \multicolumn{5}{|c|}{ Factors: $y=\frac{p_{k(a)}}{p_{z(a)}} ; b=\frac{p_{a}}{p_{k(a)}}$} \\
\hline $\begin{array}{c}\text { Work } \\
\text { conditions }\end{array}$ & $k_{1}$ & $k_{2}$ & $k_{3}$ \\
\hline I & $\frac{4.8-\frac{\kappa+1}{\kappa-1}\left(1-y^{\frac{\kappa-1}{\kappa}}\right)}{4.8-\frac{\kappa+1}{\kappa-1}\left(1-b^{\frac{\kappa-1}{\kappa}}\right)}$ & $p_{z(a)}\left(\frac{p_{a}}{p_{k(a)}}\right)^{3}$ & $\frac{\alpha_{p}}{\alpha_{w}}=1$ \\
\hline III & $\frac{\kappa}{\kappa+1}\left(\frac{2}{\kappa+1}\right)^{\frac{2}{\kappa-1}}\left(1-\frac{\lambda^{2}}{4.85}\right)$ & $\left(\frac{p_{z(a)}}{p_{k(a)}}\right)^{\frac{1}{\kappa}}$ & $\frac{\alpha_{p}}{\alpha_{w}}=1$ \\
\hline
\end{tabular}

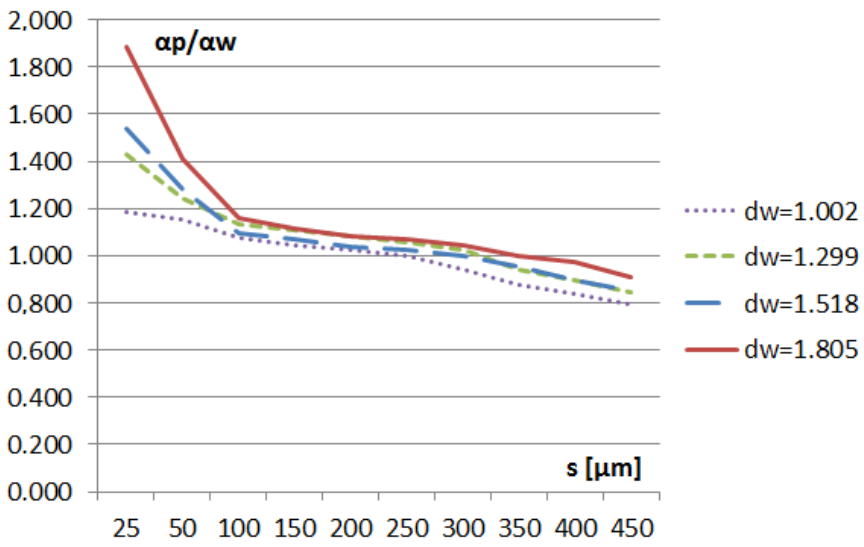

Fig. 5. The graphs of the $\alpha_{p} / \alpha_{w}$ ratio dependent on the inlet nozzle diameter $d_{w}$

Table 3. 
6. the investigated air gauge (fixed on the moving table),

7. flapper surface (not movable),

8. computer with dedicated software to control the moving table and to register its displacement and the back-pressure signal.

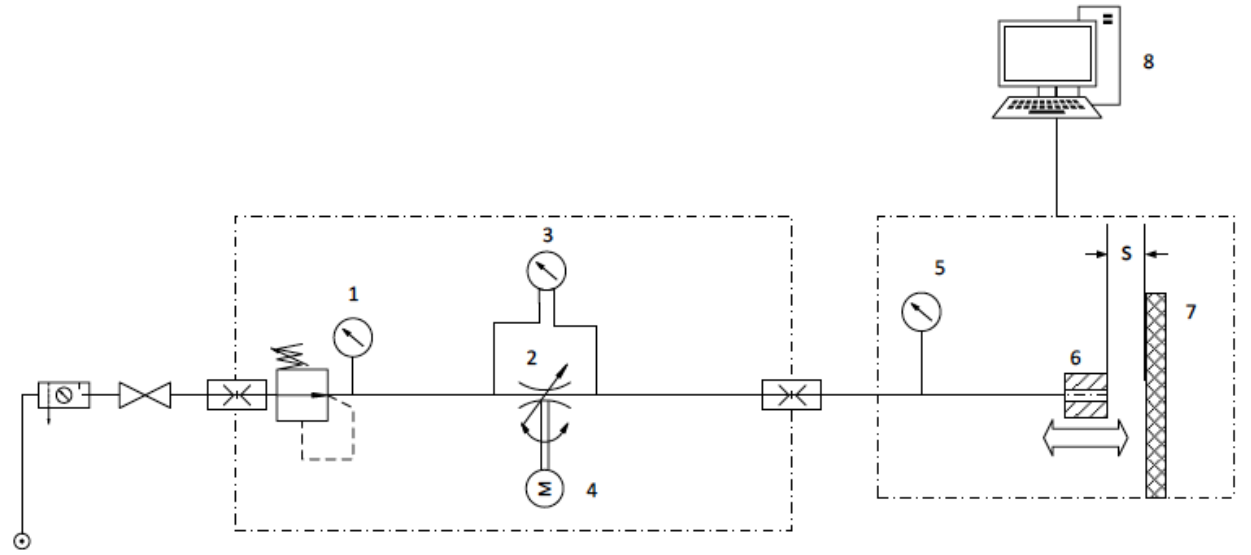

Fig. 6. Laboratory equipment for the air gauge static characteristics assessment: 1 - pressure reducer, 2 - pressure stabilizer, 3 - electronic manometer, 4 - stepper motor, 5 - pressure transducer, 6 - investigated air gauge, 7 - flapper surface, 8 - PC

The whole set is controlled by the PC equipped with program StanBad. The initial and final slot is the input, the sampling step may be chosen, the number of repetitions may be given. After the data is collected, it is processed with another program CharStat, which presents the results of the measurement. Besides the graph of pressure $p_{k}$ versus clearance $s$, the program gives a number of characteristics, like initial and final clearance $s$ for various measuring ranges $z_{p}$. Each proposed $z_{p}$ is characterized by sensitivity $K \approx \Delta p_{k} / \Delta s$, the central point and the linearity error. The latter is calculated as a mean $(S r B l)$ and the maximal value $(\mathrm{MaxBl})$ [15]:

$$
S r B l=\frac{2 \sqrt{\frac{1}{n-1} \sum_{i=1}^{n}\left(p_{k i}-\hat{p}_{k i}\right)^{2}}}{p_{k \max }-p_{k \min }} \times 100 \%
$$

where: $p_{k i}$ - the value of air pressure in the measuring chamber, measured for certain clearance $s_{i}, \hat{p}_{k i}=a_{0}+a_{1} s_{i}$ - calculated value of measuring pressure for the same clearance $s_{i}$, the coefficients of estimated linear function are $a_{0}$ and $a_{1}, p_{k \max }$ and $p_{k \text { min }}$ - the values of maximal and minimal measuring pressure for proposed measuring range $z_{p}$.

$$
M a x B l=\frac{\left|\Delta p_{k i \max }\right|}{p_{k \max }-p_{k \min }} \times 100 \%
$$


where: $\Delta p_{k i \max }$ is the maximal value of calculated and measured pressure difference: $\Delta p_{k i}=p_{k i}-\hat{p}_{k i}$.

The uncertainty of static characteristics determination was estimated as the type A uncertainty according the Guide [26] for the air gauge of multiplication $K=$ $0.525 \mathrm{kPa} / \mu \mathrm{m}$, and measuring range $z_{p}=81 \mu \mathrm{m}$. After repetitions of measurement, the beginning, the center point and the end of the proportional (linear) area of static characteristics was considered, i.e. $s_{\min }=36 \mu \mathrm{m}, s_{\text {mid }}=75 \mu \mathrm{m}$ and $s_{\max }=117 \mu \mathrm{m}$. The respective extended uncertainties on the confidence level 0.95 are as following:

$$
\begin{aligned}
& U_{0.95(p)}= \pm 0.196 \mu \mathrm{m}, \\
& U_{0.95(\text { mid })}= \pm 0.263 \mu \mathrm{m}, \\
& U_{0.95(k)}= \pm 0.176 \mu \mathrm{m} .
\end{aligned}
$$

\section{Discussion of the obtained results}

Many years of experimental investigations enabled us to work out an SCP algorithm to calculate the exact energy losses in the flow-through elements of the pneumatic cascade. The SCP algorithm is based on the second critical parameters described in details in [27], and it contains several options that make it possible:

- to choose the dimensions and shape of the nozzles (inlet and outlet ones),

- to choose from the embedded catalogue the nozzles with known second critical parameters $\alpha_{k r 2}$ and $\beta_{k r 2}$,

- to choose the way of calculation based on the back-pressure pk or the slot width s (which means that either back-pressure $p_{k}$ or slot width $s$ will be assigned with the values of constant step from the highest pressure $p_{k}=$ $p_{z}=p_{\max }$ down to $p_{k}=p_{a}$, or from the smallest slot $s=0$ up to $s=s_{\max }$ ).

The values of $\alpha_{k r 2}$ and $\beta_{k r 2}$ for various nozzles were previously determined according to the procedures described in [27] and input into the JPT program library. An example of the second critical parameters is shown in Table 5. Here, the parameters for simple cylindrical inlet nozzles are marked as (w), and the ones for the flapper-nozzle area are marked (ps). It can be seen that the outer diameter $\mathrm{dc}$ of the measuring nozzle has a substantial impact on $\alpha_{k r 2}$ and $\beta_{k r 2}$, namely, increasing their values by about $1 \%$ for $d_{c} / d_{p}=4$ compared to $d_{c} / d_{p}=2$.

Table 5.

Second critical parameters for the chosen nozzles

\begin{tabular}{|c|c|c|c|c|c|c|}
\hline & \multicolumn{3}{|c|}{$d_{c} / d_{p}=2$} & \multicolumn{3}{c|}{$d_{c} / d_{p}=4$} \\
\hline & $\begin{array}{c}d_{p}=1.518 \\
{[\mathrm{~mm}]}\end{array}$ & $\begin{array}{c}d_{w}=1.002 \\
{[\mathrm{~mm}]}\end{array}$ & $\begin{array}{c}d_{w}=1.299 \\
{[\mathrm{~mm}]}\end{array}$ & $\begin{array}{c}d_{p}=1.518 \\
{[\mathrm{~mm}]}\end{array}$ & $\begin{array}{c}d_{w}=1.002 \\
{[\mathrm{~mm}]}\end{array}$ & $\begin{array}{c}d_{w}=1.299 \\
{[\mathrm{~mm}]}\end{array}$ \\
\hline$\alpha_{k r 2}(\mathrm{w})$ & & 0.879 & 0.843 & & 0.879 & 0.843 \\
$\beta_{k r 2}(\mathrm{w})$ & & 0.467 & 0.478 & & 0.467 & 0.478 \\
\hline$\alpha_{k r 2}(\mathrm{ps})$ & 0.861 & & & 0.892 & & \\
$\beta_{k r 2}(\mathrm{ps})$ & 0.513 & & & 0.518 & & \\
\hline
\end{tabular}


In the program, there are also options to choose different conditions of the feeding airflow as well as the environmental conditions. In this way, the real industrial work conditions could be simulated as closely as possible. Moreover, the gas other than air could be considered, too, which may provide some improvement of the measurement accuracy.

The simulation provides the static characteristics that are very close to the empirical ones. In Figs 7 and 8, there are presented the graphs of the approximation error $\delta[\%]$ between the experimental static characteristics and the ones simulated with second critical parameters (SCP) method. To emphasize the accuracy of the SCP method, the other functions are added. The graphs of approximation error $\delta$ [\%] between the actual and theoretical static characteristics are presented in Fig. 7. The curves represent the following functions: "Saint Venant-Wenzel" - Table 1, "simplified" - Table 2, "Balakszin" - Table 3, "Gluchov" - Table 4 (for $\alpha=1$ ), "Gluchov mod." - Table 4 (for $\alpha \neq 1$ ).

The graphs reveal different values of the error $\delta$ for different slot widths $s$. In fact, only Saint Venant-Wenzel function either in full or in simplified form provide acceptable accuracy in a large extend (for $s$ up to 200 or almost $300 \mu \mathrm{m}$ ). Hence, it could be assumed that there are two main sources of generated errors: calculations are made $\left(1^{\text {st }}\right)$ for non-compressible flow and $\left(2^{\text {nd }}\right)$ for the arbitrarily approximated values of flow coefficients $\alpha_{w}, \alpha_{p}$. In that case, the true pressure losses are not taken into account. The approximation error $\delta$ [\%] is calculated using equation:

$$
\delta=\frac{p_{k(\exp )}}{p_{k(\operatorname{sim})}} \times 100 \%
$$

where $p_{k(\exp )}$ is experimental back-pressure result, $p_{k(\text { sim })}$ is simulated back-pressure result.

It is clearly seen that the second critical parameter (SCP) method provides much better approximation of the static characteristics which differ from the experimental ones not more than by $5 \%$. In the proportional area, which is used as a measuring rang of the air gauge, the error is even smaller. For example, the analysis of the sensitivity $|K|$ related to $\left|K_{\max }\right|$ (Fig. 9) shows that the measuring ranges are different for different outer diameters $d_{c}$. Table 6 presents the values of the smallest $\left(s_{\min }\right)$, middle $\left(s_{\text {mid }}\right.$ which correspond to maximal sensitivity $\left.\left|K_{\max }\right|\right)$ and the largest $\left(s_{\max }\right)$ measuring slots of the proportional area (measuring range $\left.z_{p}\right)$.

Table 6. Measuring range $z_{p}$ characteristics of the air gauge $\left(d_{p}=1.518 \mathrm{~mm}, d_{w}=1.299 \mathrm{~mm}\right)$ of different outer diameter ratios $D_{c}=d_{c} / d_{p}$

\begin{tabular}{|l|c|c|c|}
\hline$z_{p}$ characteristics & $D_{c}=2$ & $D_{c}=3$ & $D_{c}=4$ \\
\hline$s_{\min }[\mu \mathrm{m}]$ & 146 & 140 & 137 \\
\hline$s_{\operatorname{mid}}[\mu \mathrm{m}]\left(\right.$ for $\left.K_{\max }\right)$ & 200 & 188 & 185 \\
\hline$s_{\max }[\mu \mathrm{m}]$ & 217 & 237 & 253 \\
\hline
\end{tabular}




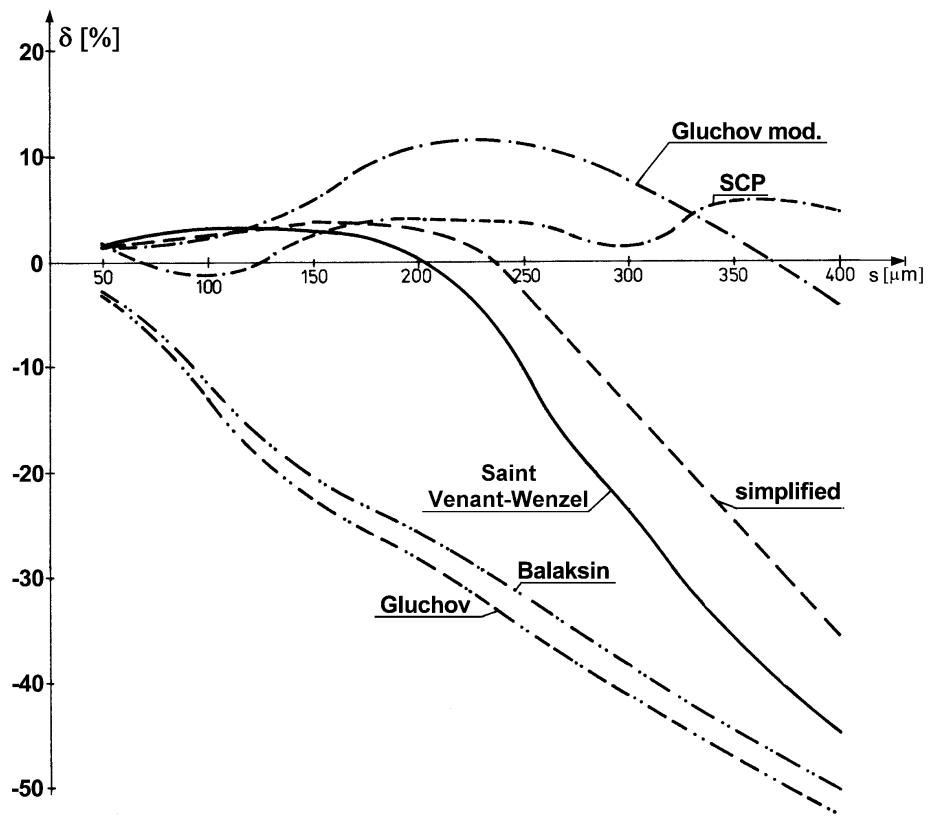

(a)

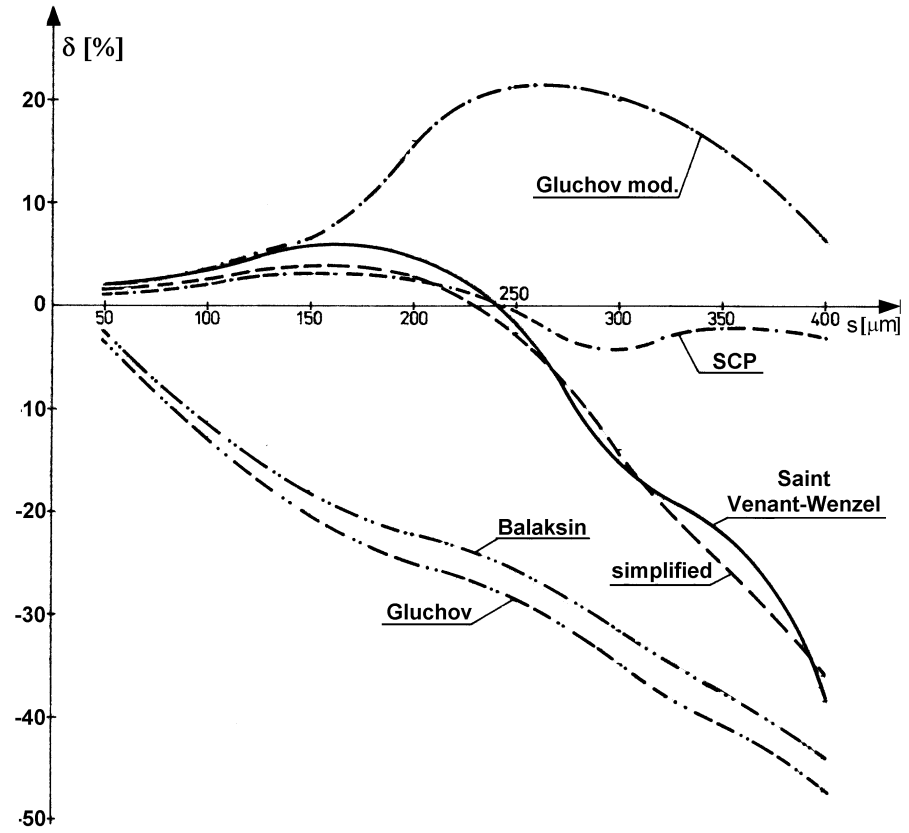

(b)

Fig. 7. Differences $\delta$ between the actual and simulated static characteristics of the air gauge of $d_{p}=1.518 \mathrm{~mm}$ with inlet nozzle $d_{w}=1.002 \mathrm{~mm}$ for different outer diameters: a) $d_{c} / d_{p}=2$, b) $d_{c} / d_{p}=4$ 


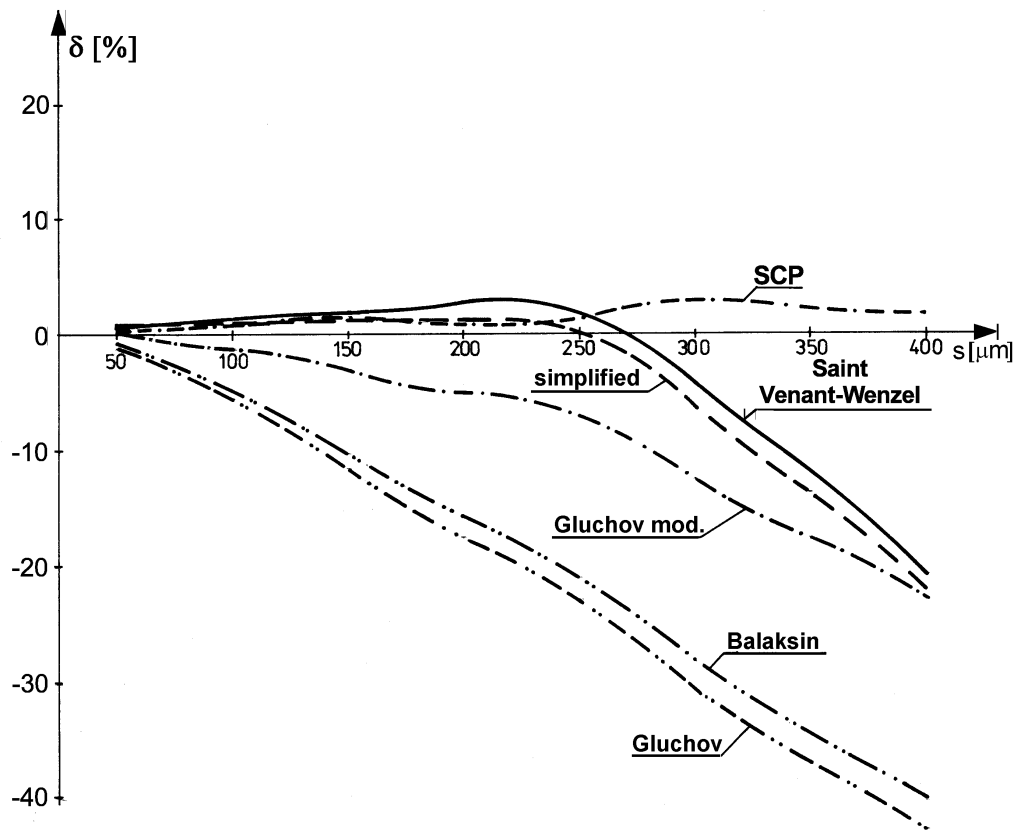

(a)

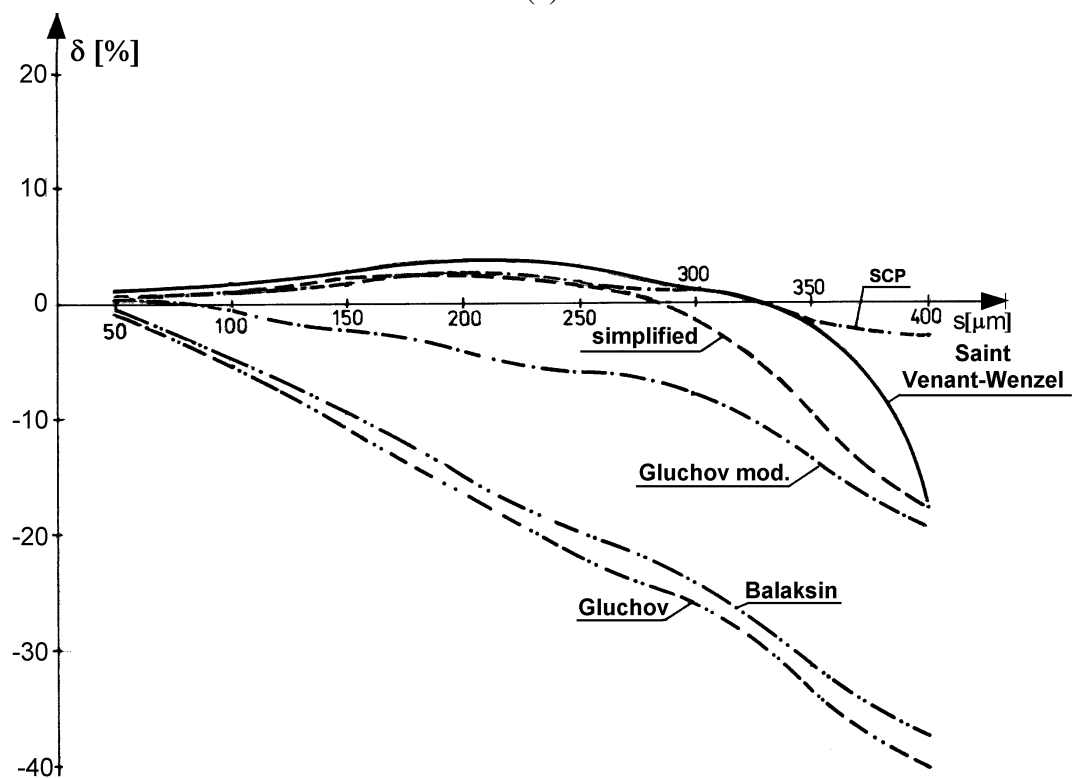

(b)

Fig. 8. Differences $\delta$ between the actual and simulated static characteristics of the air gauge of $d_{p}=1.518 \mathrm{~mm}$ with inlet nozzle $d_{w}=1.299 \mathrm{~mm}$ for different outer diameters: a) $d_{c} / d_{p}=2$, b) $d_{c} / d_{p}=4$ 


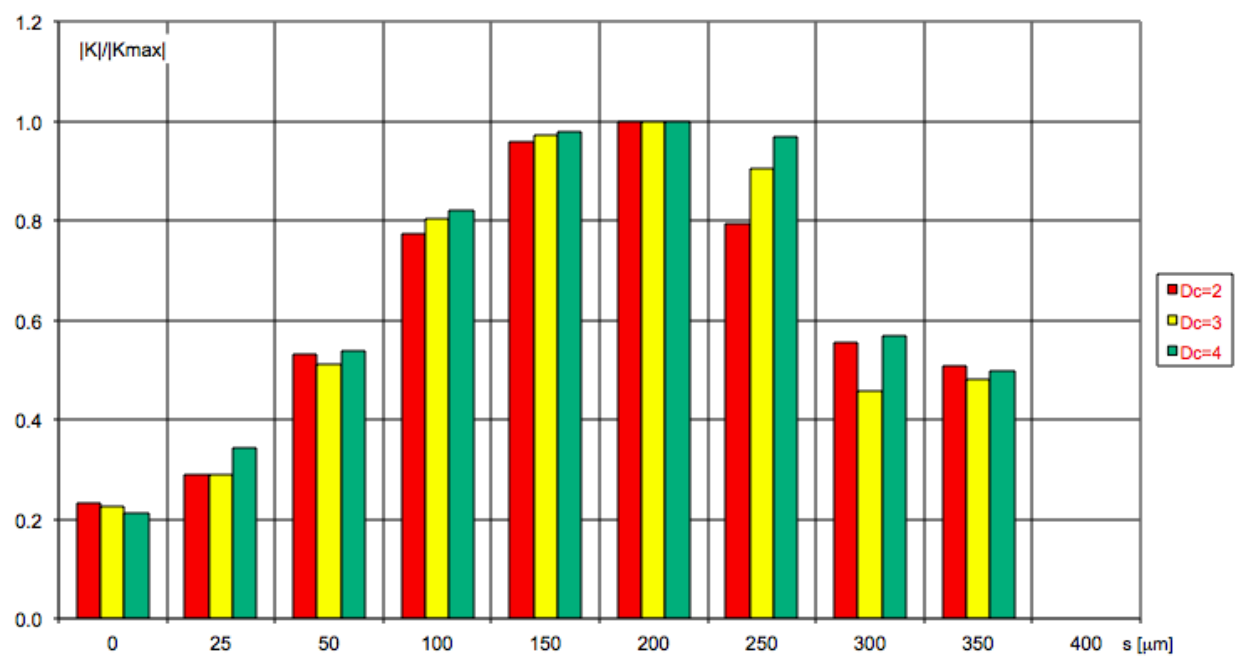

Fig. 9. The normalized sensitivity $\mathrm{K}$ for static characteristic of the air gauge $\left(d_{p}=1.518 \mathrm{~mm}\right.$, $\left.d_{w}=1.299 \mathrm{~mm}\right)$ of different outer diameter ratios $D_{c}=d_{c} / d_{p}(2,3$ and 4$)$

Fig.10 illustrates how the approximation with different functions could fit the measuring range $z_{p}$.

In the example presented in Fig. 10, the approximation errors take the smallest values at the beginning of the measuring range $\left(s_{\min }\right)$ for all methods. The simplified Saint Venant formulas and the SPC method provide quite similar results with the errors $\delta \approx 1 \%$. However, the SPC error is much smaller for all slots from $s=0$ up to $s=400 \mu \mathrm{m}$, which is important in initial calculations, before the exact measuring range is known.

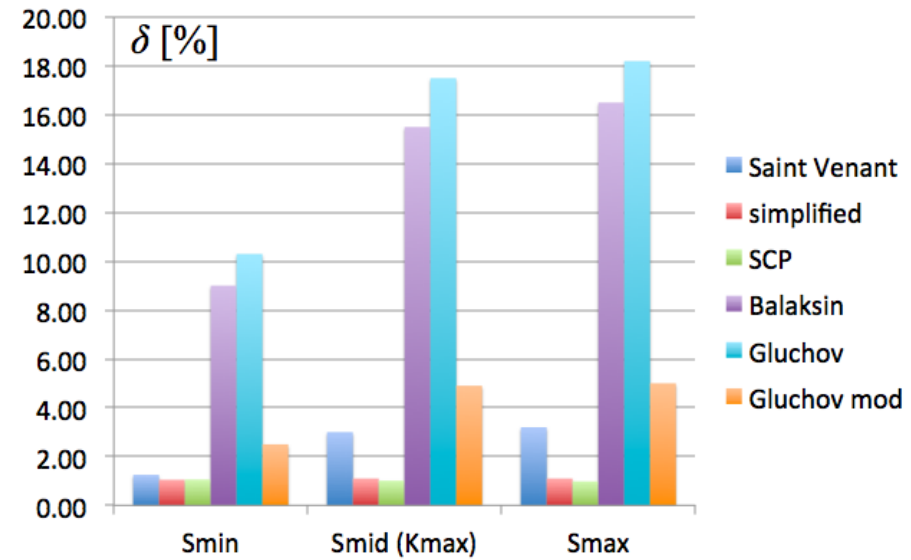

Fig. 10. Approximation error $\delta[\%]$ related to the measuring range $z_{p}$ (the air gauge: $d_{p}=1.518$ $\left.\mathrm{mm}, D_{c}=d_{c} / d_{p}=2, d_{w}=1.299 \mathrm{~mm}\right)$ 
This is not the case also for other sets of nozzles. For example, SPC method keeps the approximation error close to $1 \%$ almost through all the measuring range, while the Saint Venant formulas (both exact and simplified) provide the approximation with $\delta \approx 3 \%$ (Fig. 11).

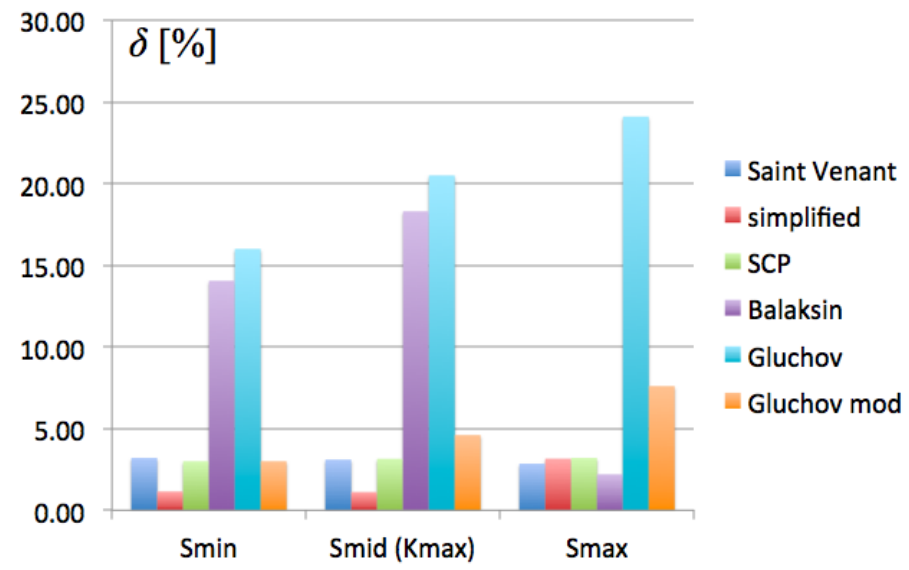

Fig. 11. Approximation error $\delta[\%]$ related to the measuring range $z_{p}$ (the air gauge: $d_{p}=1.518$ $\left.\mathrm{mm}, D_{c}=d_{c} / d_{p}=2, d_{w}=1.002 \mathrm{~mm}\right)$

\section{Conclusions}

Even though the principle of air gauging is known for a hundred years now, there is still some room for improvement of this excellent measuring tool [28]. Its accuracy went better so much that it became necessary to replace the commonly known estimation methods with more precise ones. The SCP method based on the so-called second critical parameters (flow coefficient $\alpha_{k r 2}$ and pressure ratio $\beta_{k r 2}$ that describe the true air flow through the air gauge elements) proved the ability to provide the estimation on the satisfactory level of $3 \%$ error (maximally $5 \%$ in the areas out of the measuring range). Easy-to-use and equipped with necessary dataset program allows one to calculate a wide range of characteristics for various nozzle couples with the known orifice geometry.

\section{Acknowledgements}

The investigation described in this work were supported by the Ministry of Science and Higher Education, Poland. 


\section{References}

[1] M. Mennesson. High precision measurement method of lengths and thicknesses. Comptes Rendus des Seances de l'Academie des Sciences, 194(25.4.1932):1459-1461, 1932.

[2] W.J. Gluchow and A.A. Tupolew. Non-contact pneumatic measuring control devices for the production of workpieces with discontinuous surfaces. Feingerätetechnik, 23(2):69-73, 1974. (in German).

[3] E.I. Ped. Optimization of the constructional elements choice of the air gauges designed for the dynamic measurements. Measurement Techniques, 7:29-31, 1981. (in Russian).

[4] F.T. Farago and Curtis M.A. Handbook of Dimensional Measurement. Industrial Press Inc., New York, 1994.

[5] G. Schuetz. Pushing the limits of air gaging-and keeping them there. Quality, 54(7):22-26, 2015.

[6] G. Schuetz. Air gaging gets better with age. Quality, 3:28-32, 2008.

[7] L. Finkelstein. Reflections on a century of measurement science as an academic discipline. Metrology and Measurement Systems, 14(4):635-638, 2007.

[8] M. Rucki, B. Barisic, and G. Varga. Air gauges as a part of the dimensional inspection systems. Measurement, 43(1):83-91, 2010. doi: 10.1016/j.measurement.2009.07.001.

[9] T. Janiczek and J. Janiczek. Linear dynamic system identification in the frequency domain using fractional derivatives. Metrology and Measurement Systems, 17(2):279-288, 2010. 10.2478/v10178-010-0024-6.

[10] V.B. Bokov. Pneumatic gauge steady-state modelling by theoretical and empirical methods. Measurement, 44(2):303-311, 2011. doi: 10.1016/j.measurement.2009.01.015.

[11] B. Dobrowolski, Z. Kabza, and A. Spyra. Digital simulation of air flow through a nozzle of pneumatic gauge. In Proc. 33rd Annual Conference JUREMA, pages 67-70, 1988.

[12] M.N. Abhari, M. Ghodsian, M. Vaghefi, and N. Panahpur. Experimental and numerical simulation of flow in a $90^{\circ}$ bend. Flow Measurement and Instrumentation, 21(3):292-298, 2010. doi: 10.1016/j.flowmeasinst.2010.03.002.

[13] J. Peng, X. Fu, and Y. Chen. Response of a swirlmeter to oscillatory flow. Flow Measurement and Instrumentation, 19(2):107-115, 2008. doi: 10.1016/j.flowmeasinst.2007.10.002.

[14] C. Crnojevic, G. Roy, A. Bettahar, and P. Florent. The influence of the regulator diameter and injection nozzle geometry on the flow structure in pneumatic dimensional control systems. Journal of Fluids Engineering, 119:609-615, 1997. doi: 10.1115/1.2819288.

[15] C. Jermak, editor. Theory and Practice of Air Gauging. Poznan University of Technology, 2011.

[16] T. Kiczkowiak and S. Grymek. Critical pressure ratio b as defined in iso 6358 and iso 6953 standards. Pomiary Automatyka Kontrola (Measurement, Automation, Monitoring), 57:559562, 2011. (in Polish).

[17] A Cellary and C.J. Jermak. Dynamics of a cascade pneumatic sensor for length measurements. In Proc. of Optoelectronic and Electronic Sensors II, pages 36-39. International Society for Optics and Photonics, 1997. doi: 10.1117/12.266719.

[18] A.V. Deych. Technical gasodynamics. Gosenergoizdat, Moscow, 1961. (in Russian).

[19] M. Kabacinski, C. T Lachowicz, and J. Pospolita. Numerical analysis of flow averaging tubes in the vortex-shedding regime. Archive of Mechanical Engineering, 60(2):283-297, 2013. doi: 10.2478/meceng-2013-0018.

[20] Kościelny W. and Woźniak C. Models of the flow characteristics of the pneumatic restrictors. In Proc. PNEUMA'95, pages 73-82, 1995. (in Polish).

[21] Kościelny W. and Woźniak C. Experimental evaluation of the models of the pneumatic restrictors flow characteristics. In Proc. PNEUMA'95, pages 83-92, 1995. (in Polish). 
[22] Automation of the pneumatic dimensional measurement in mechanical engineering. Mashinostroyeniye, Moscow, 1964. (in Russian).

[23] C.J. Jermak. Methods of shaping the metrological characteristics of air gages. Strojniski Vestnik/Journal of Mechanical Engineering, 56(6):385-390, 2010.

[24] R.J. Soboczyński. Investigations on the metrological properties of high pressure air gauges. $\mathrm{PhD}$ thesis, Wrocław Technical University, 1977. (in Polish).

[25] Calculation of the high pressure air gauges characteristics. Journal Measuring Techniques, 6:107, 1971.

[26] Guide to the expression of uncertainty in measurement. Warszawa, Główny Urząd Miar, 1999. (in Polish).

[27] C.J. Jermak and M. Rucki. Air gauging: Static and dynamic characteristics. IFSA, Barcelona, Spain, 2012.

[28] Cz.J. Jermak and M. Rucki. Air gauging: Still some room for development. AASCIT Communication, 2(2):29-34, 2015. 